\title{
Prevalência de dor neuropática em pacientes com fratura da extremidade distal do rádio tratada com placa volar bloqueada
}

\section{Prevalence of Neuropathic Pain in Patients with Fracture of the Distal Extremity of the Radius Treated with Volar Locking Plate}

\author{
Helbert Luiz Nomura da Silva ${ }^{1}$ Giuliana Olivi Tanaka ${ }^{2}$ Thiago Barros Pinheiro ${ }^{2}$ Yussef Ali Abdouni ${ }^{3}$
}

\footnotetext{
${ }^{1}$ Médico Ex-residente do Grupo de Cirurgia da Mão e Microcirurgia, Faculdade de Ciências Médicas da Santa Casa de São Paulo, São Paulo, SP, Brasil

2 Médico Residente de Ortopedia e Traumatologia do Departamento de Ortopedia e Traumatologia, Faculdade de Ciências Médicas da Santa Casa de São Paulo, São Paulo, SP, Brasil

${ }^{3}$ Médico Assistente do Grupo de Cirurgia da Mão e Microcirurgia, Faculdade de Ciências Médicas da Santa Casa de São Paulo, São Paulo, SP, Brasil
}

Rev Bras Ortop 2022;57(6):924-929.
Endereço para correspondência Giuliana Olivi Tanaka, MD, Santa Casa de Misericórdia de São Paulo, Departamento de Ortopedia e Traumatologia “Pavilhão Fernandinho Simonsen”, Rua Doutor Cesário Mota Júnior, 112-Vila Buarque, 01221-020. São Paulo, SP, Brasil (e-mail: olivitkg@gmail.com).

\begin{abstract}
Resumo
Palavras-chave

- fratura do rádio

- medição da dor

- complicações pósoperatórias

- fixação interna de fraturas

Objetivo Avaliar a prevalência de dor persistente no pós-operatório de fratura da extremidade distal do rádio, assim como detectar precocemente sinais de dor neuropática com o intuito de desenvolver protocolos de prevenção da dor crônica pós-operatória. Métodos Estudo prospectivo, realizado com 56 pacientes submetidos a redução aberta e fixação interna de fratura da extremidade distal do rádio com placa volar bloqueada no período de março a setembro de 2020. Os pacientes foram submetidos a avaliação de dor neuropática e capacidade funcional através dos questionários Douleur Neuropathique 4 questionnaire (DN4) e Quick Disabilities of the Arm, Shoulder, and Hand (Quick-DASH, na sigla em inglês). As variáveis qualitativas foram comparadas pelo teste de Mann-Whitney e sua correlação foi analisada pelos testes de correlação de Spearman e de Igualdade de Duas Proporções.
\end{abstract}

Trabalho desenvolvido no Grupo de Cirurgia da Mão e Microcirurgia do Departamento de Ortopedia e Traumatologia da Faculdade de Ciências Médicas da Santa Casa de São Paulo, "Pavilhão Fernandinho Simonsen" (DOT - FCMSCSP) (Diretora: Professora Doutora Maria Fernanada Silber Caffaro), São Paulo, SP, Brasil. recebido

04 de Abril de 2021

aceito

15 de Junho de 2021

Publicado on-line

de Janeiro 21, 2022
DOI https://doi.org/

$10.1055 / \mathrm{s}-0041-1736526$. ISSN 0102-3616.

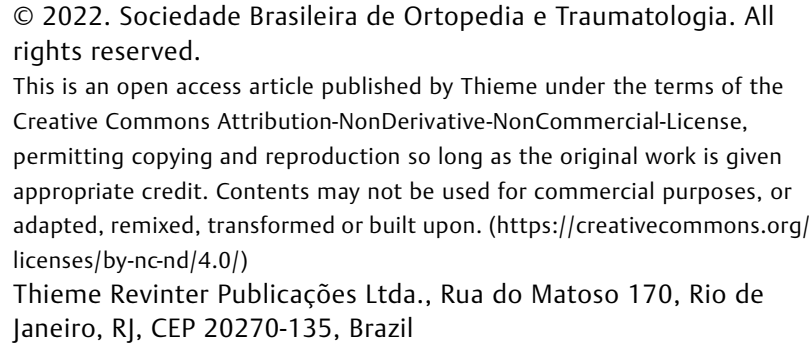

(c) 2022. Sociedade Brasileira de Ortopedia e Traumatologia. All rights reserved.

This is an open access article published by Thieme under the terms of the Creative Commons Attribution-NonDerivative-NonCommercial-License, permitting copying and reproduction so long as the original work is given appropriate credit. Contents may not be used for commercial purposes, or adapted, remixed, transformed or built upon. (https://creativecommons.org/ licenses/by-nc-nd/4.0/) Thieme Revinter Publicações Ltda., Rua do Matoso 170, Rio de Janeiro, RJ, CEP 20270-135, Brazil 


\section{Abstract}

\section{Keywords}

- radius fractures

- pain measurement

- postoperative complications

- fracture fixation, internal
Resultados Foram incluídos no presente estudo 43 pacientes, com idades entre 18 e 66 anos; 39,5\% dos participantes apresentaram pontuação $\geq 4$ no questionário Douleur neuropathique 4 questionnaire (DN4). Em relação ao Quick-DASH, a média foi de 38,6 . Não houve diferença estatística significante entre o sexo do paciente e o valor do DN4 $(p=0,921)$. Também não foi encontrada correlação estatística entre as variáveis quantitativas DN4 e Quick-DASH $(p=0,061)$.

Conclusões A prevalência de dor neuropática nos pacientes pós-operatórios analisados foi significativa e a presença de sinais e sintomas de dor neuropática foi fator preditivo positivo para a persistência da dor além de 2 meses em 100\% dos casos. Assim, com diagnóstico precoce do componente neuropático de dor, associado ao componente nociceptivo, é possível obter o controle adequado da dor, impedindo sua cronificação e garantindo uma melhor reabilitação.

Objective To evaluate the prevalence of persistent pain in the postoperative period of fractures of the distal extremity of the radius, as well as to detect early signs of neuropathic pain to develop protocols for the prevention of chronic postoperative pain. Methods Prospective study, carried out with 56 patients who underwent open reduction and internal fixation of fractures of the distal extremity of the radius with a volar locking plate from March to September 2020. The patients were submitted to assessment of neuropathic pain and functional capacity through the Douleur Neuropathique 4 questionnaire (DN4) and Quick Disabilities of the Arm, Shoulder, and Hand (Quick-DASH) questionnaires. Qualitative variables were compared using the MannWhitney $\mathrm{U}$ test and their correlation was analyzed using the Spearman Correlation and Equality of Two Proportions tests.

Results A total of 43 patients aged between 18 and 66 years old were included in the present study; $39.5 \%$ of the participants scored $\geq 4$ on the DN4 questionnaire. In relation to Quick-DASH, the average was 38.6 . There was no statistically significant difference between the gender of the patient and the DN4 value $(p=0.921)$. There was also no statistical correlation between the quantitative variables DN4 and Quick-DASH $(p=0.061)$.

Conclusions The prevalence of neuropathic pain in analyzed postoperative patients was significant, and the presence of signs and symptoms of neuropathic pain was a positive predictive factor for pain persistence beyond 2 months in $100 \%$ of cases. Thus, with early diagnosis of the neuropathic component of pain, associated with the nociceptive component, adequate pain control can be achieved, preventing its chronicity, and ensuring better rehabilitation.

\section{Introdução}

As fraturas na extremidade distal do antebraço são as mais frequentes no membro superior, correspondendo a $74 \%$ das fraturas dos ossos do antebraço e a $16 \%$ de todas as fraturas do esqueleto. ${ }^{1}$ Tais fraturas podem ser decorrentes de traumatismos de alta energia, geralmente em pacientes jovens, ou de traumatismos de baixa energia em pacientes idosos, portadores de osteoporose. Quando essas fraturas são irredutíveis ou instáveis, o tratamento cirúrgico é o indicado. Com a evolução dos implantes, em especial das placas com bloqueio para a extremidade distal do rádio, tornou-se possível o reestabelecimento da anatomia da região, com fixação estável que permite mobilidade precoce. $^{2}$ No entanto, apesar dos excelentes resultados radiográficos obtidos com a redução aberta e fixação interna destas fraturas com placa volar bloqueada, clinicamente os resultados não são homogêneos.

Apesar de ainda pouco documentada na literatura, a dor crônica pós-operatória (DCPO) tem sido descrita como um dos principais fatores que interferem na reabilitação dos pacientes submetidos a cirurgias ortopédicas, afetando, assim, sua capacidade laboral e sua qualidade de vida.

A DCPO é definida como aquela que ocorre após procedimento cirúrgico, com pelo menos 2 meses de duração, não estando relacionada com a dor pré-existente à cirurgia e sem outras etiologias definidas, como infecção, por exemplo. ${ }^{3}$ Sua 


\section{ENTREVISTA DO PACIENTE \\ Questão 1: A sua dor tem uma ou mais das seguintes características ? \\ 1. Queimação \\ 2. Sensação de frio dolorosa \\ 3. Choque elétrico} Questão 2: Há presença de um ou mais dos seguintes sintomas na mesma área da sua
dor?

4. Formigamento

5. Alfinetada e agulhada

6. Adormecimento

7. Coceira

EXAME DO PACIENTE
Questão 3 A dor esta localizada numa área onde o exame fisico pode revelar uma ou
mais das seguintes caracteristicas?
8. Hipoestesia ao toque
9. Hipoestesia a picada de agulha

Questão 4: Na área dolorosa a dor pode ser causada ou aumentada por:

10. Escovação

$$
\operatorname{Sim}=1 \text { ponto Não }=\text { zero ponto }
$$

Fig. 1 Questionário DN4 para diagnóstico de dor neuropática. Fonte: Santos et al. ${ }^{7}$

incidência é muito variável, ocorrendo em entre 5 e $80 \%$ dos pacientes submetidos a procedimentos cirúrgicos de diversos tipos. ${ }^{4}$ Esta grande variação pode estar associada a falhas na avaliação e, consequentemente, ao diagnóstico desta entidade.

No período pós-operatório imediato, a ativação direta de nociceptores, a resposta inflamatória e a eventual lesão de estruturas nervosas provocam, do ponto de vista clínico, dor em repouso no local da cirurgia e em região próxima. Há também dor desencadeada pelo toque ou pelo movimento, indicando sensibilização periférica.

Um componente neuropático pode se desenvolver imediatamente após o trauma cirúrgico e persistir na ausência de estímulo periférico nociceptivo ou inflamatório. Deste modo, definir dor neuropática é essencial para elaborar estratégias de prevenção e tratamento da dor crônica persistente. ${ }^{5,6}$ O questionário Douleur Neuropathique 4 questionnaire (DN4), ${ }^{7}$ traduzido e validado para o português, tem sido largamente empregado como instrumento de rastreio da dor neuropática, por ser de fácil aplicação tanto por especialistas em dor quanto por não especialistas. O DN4 é composto por sete itens que se referem a sintomas e outros três que se relacionam com o exame físico. Cada item pontua 1 se a resposta for positiva e zero se negativa, levando a um valor mínimo de zero e o máximo de 10 . A somatória de pontos $\geq 4$ sugere dor neuropática.

O objetivo do presente estudo é avaliar a prevalência de dor persistente no pós-operatório de fratura da extremidade distal do rádio, assim como detectar precocemente sinais de dor neuropática, com o intuito de desenvolver protocolos de prevenção da DCPO.

\section{Material e Métodos}

Foram avaliados prospectivamente 56 pacientes, com idade mínima de 18 anos, apresentando fratura na extremidade distal do rádio, atendidos em nossa instituição e submetidos a redução aberta e fixação interna com placa volar bloqueada, no período de março a setembro de 2020. Foram critérios de exclusão neste estudo pacientes com fraturas expostas, fraturas bilaterais, com lesões nervosas associadas ou ainda aqueles que apresentaram infecção do sítio cirúrgico.

Após 10 semanas da cirurgia, cada paciente era submetido à avaliação de dor neuropática através do questionário DN4 (-Figura 1) em sua versão traduzida e validada para 0 português. Neste momento, foi aplicado também o questionário Quick-DASH ( - Tabela 1), igualmente traduzido para o português, para avaliar o impacto funcional sobre o membro acometido. O Quick-DASH é um questionário específico que avalia o nível de incapacidade em pacientes com várias desordens do membro superior, associando o sintoma e seu impacto na disfunção de atividades físicas, sociais e psicológicas. A pontuação é apresentada em uma escala de orientação positiva de 0 (máxima funcionalidade) a 100 (máxima incapacidade), incluindo 2 módulos opcionais (Módulo trabalho e Módulo esporte/música) com 4 itens cada (1 a 5). ${ }^{8}$ Ambos os questionários foram aplicados sempre pelo mesmo avaliador.

As variáveis qualitativas foram comparadas pelo teste de Mann-Whitney e sua correlação foi analisada pelos testes de Correlação de Spearman e de Igualdade de Duas Proporções. Foram utilizados os softwares Excel Office 2010 (Microsoft Corporation, Redmond WA, EUA), IBM SPSS Statistics for Windows version 20.0 (IBM Corp., Armonk, NY, EUA) e 
Tabela 1 Questionário QuickDASH

\begin{tabular}{|c|c|c|c|c|c|}
\hline & $\begin{array}{l}\text { Não houve } \\
\text { dificuldade }\end{array}$ & $\begin{array}{l}\text { Houve pouca } \\
\text { dificuldade }\end{array}$ & $\begin{array}{l}\text { Houve dificuldade } \\
\text { moderada }\end{array}$ & $\begin{array}{l}\text { Dificuldade } \\
\text { severa }\end{array}$ & $\begin{array}{l}\text { Não } \\
\text { conseguiu fazer }\end{array}$ \\
\hline 1.Abrir um vidro novo ou com tampa muito apertada. & 1 & 2 & 3 & 4 & 5 \\
\hline $\begin{array}{l}\text { 2.Fazer tarefas domésticas pesadas } \\
\text { (por exemplo: lavar paredes, lavar o chão). }\end{array}$ & 1 & 2 & 3 & 4 & 5 \\
\hline 3.Carregar uma sacola ou uma maleta. & 1 & 2 & 3 & 4 & 5 \\
\hline 4.Lavar suas costas. & 1 & 2 & 3 & 4 & 5 \\
\hline 5.Usar uma faca para cortar alimentos. & 1 & 2 & 3 & 4 & 5 \\
\hline \multirow{2}{*}{$\begin{array}{l}\text { 6.Atividades recreativas que exigem alguma força ou } \\
\text { impacto nos braços, ombros ou mãos } \\
\text { (por exemplo: jogar vôlei, martelar). }\end{array}$} & 1 & 2 & 3 & 4 & 5 \\
\hline & Não afetou & Afetou pouco & $\begin{array}{l}\text { Afetou } \\
\text { moderadamente }\end{array}$ & Afetou muito & $\begin{array}{l}\text { Afetou } \\
\text { extremamente }\end{array}$ \\
\hline \multirow{2}{*}{$\begin{array}{l}\text { 7.Durante a semana passada, em que ponto } \\
\text { o seu problema com o braço, ombro ou mão } \\
\text { afetaram suas atividades normais com família, } \\
\text { amigos, vizinhos ou colegas? }\end{array}$} & 1 & 2 & 3 & 4 & 5 \\
\hline & Não limitou & Limitou Pouco & $\begin{array}{l}\text { Limitou } \\
\text { moderadamente }\end{array}$ & Limitou muito & $\begin{array}{l}\text { Não } \\
\text { conseguiu fazer }\end{array}$ \\
\hline $\begin{array}{l}\text { 8.Durante a semana passada, o seu trabalho ou outras } \\
\text { atividades diárias regulares foram limitadas } \\
\text { devido ao seu problema com braço, ombro ou mão? }\end{array}$ & 1 & 2 & 3 & 4 & 5 \\
\hline $\begin{array}{l}\text { Por favor, meça a gravidade dos seguintes } \\
\text { sintomas na semana passada. (circule o número) }\end{array}$ & Nenhuma & Pouca & Moderada & Severa & Extrema \\
\hline 9.Dor no braço, ombro ou mão & 1 & 2 & 3 & 4 & 5 \\
\hline \multirow{2}{*}{$\begin{array}{l}\text { 10.Desconforto na pele (alfinetadas) } \\
\text { no braço, ombro ou mão }\end{array}$} & 1 & 2 & 3 & 4 & 5 \\
\hline & $\begin{array}{l}\text { Não houve } \\
\text { dificuldade }\end{array}$ & $\begin{array}{l}\text { Pouca } \\
\text { dificuldade }\end{array}$ & $\begin{array}{l}\text { Dificuldade } \\
\text { moderada }\end{array}$ & $\begin{array}{l}\text { Dificuldade } \\
\text { severa }\end{array}$ & $\begin{array}{l}\text { Tão difícil } \\
\text { que eu não } \\
\text { pude dormir }\end{array}$ \\
\hline $\begin{array}{l}\text { 11.Durante a semana passada, quanto de dificuldade } \\
\text { você teve para dormir por causa da dor } \\
\text { no seu braço, ombro ou mão? (circule o número) }\end{array}$ & 1 & 2 & 3 & 4 & 5 \\
\hline
\end{tabular}

Nota: Por favor, meça sua habilidade para realizar as seguintes atividades na semana passada circulando o número apropriado da resposta.

Tabela 2 Variáveis quantitativas

\begin{tabular}{|l|l|l|l|l|l|l|}
\hline & Média & Mediana & Desvio padrão & Mínimo & Máximo & IC \\
\hline Idade & 42,3 & 42 & 14,5 & 18 & 66 & 4,3 \\
\hline DN4 & 2,88 & 3 & 1,62 & 0 & 6 & 0,48 \\
\hline Quick-DASH & 38,6 & 35 & 18,4 & 15 & 80 & 55 \\
\hline
\end{tabular}

Abreviações: DN4, Douleur Neuropathique 4 questionnaire; IC, intervalo de confiança; Quick-DASH, Quick Disabilities of the Arm, Shoulder, and Hand.

Minitab 16, (Minitab, State College, PA, EUA) e foi considerada significância estatística quando o valor de p foi $<0,05$ (5\%).

\section{Resultados}

Foram incluídos no presente estudo 43 pacientes, com idades entre 18 e 66 anos (média 42,3 anos), sendo 32 (74,4\%) do sexo masculino e 11 (25,6\%) do sexo feminino.

Os escores obtidos com a aplicação do questionário DN4 variaram de 0 a 6 , sendo que 17 pacientes (39,5\%) apresentaram pontuação $\geq 4$, a qual indica a presença de componente neuropático na dor. Em relação ao Quick-DASH, a pontuação neste questionário variou de 15 a 80, com média de 38,6 . As variáveis quantitativas estão representadas na - Tabela 2.

Após a aplicação dos testes estatísticos, foi observado que não houve diferença estatística significante entre o sexo do paciente e o valor do DN4 ( $p=0,921$ ) (-Figura 2). Também não foi encontrada correlação estatística entre as variáveis quantitativas DN4 e Quick-DASH $(p=0,061)$.

\section{Discussão}

As fraturas da extremidade distal do rádio são muito frequentes e, a despeito do tratamento adequado, é frequente a persistência de déficit funcional. Orbay et al. ${ }^{9}$ relataram que, 


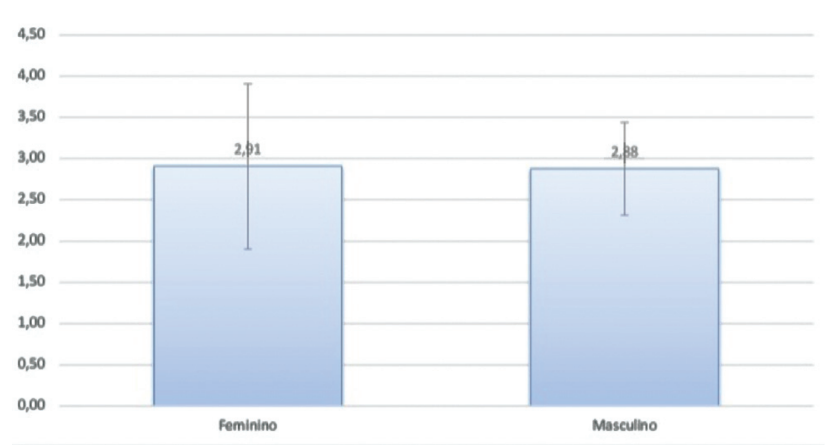

Fig. 2 Distribuição dos pacientes com DN4 $\geq 4$ por sexo.

após 1 ano de cirurgia com placa volar de ângulo fixo, persistia uma perda da força de preensão, com média de $79 \%$ em relação ao lado contralateral. Catalano et al. ${ }^{10}$ observaram discrepância significativa entre os resultados radiográficos e os resultados funcionais, sugerindo que existem mais processos em questão do que a simples redução da fratura e consolidação óssea. Um quadro de dor persistente pode ser o fator determinante para o retardo no processo de reabilitação, levando à perda da amplitude de movimento e da força no longo prazo.

Ibor et al., ${ }^{11}$ em um estudo multicêntrico com 5.024 pacientes ortopédicos, detectaram que a dor mista era a mais prevalente entre estes pacientes (59,3\%), incluindo a dor pós-operatória. Este estudo observou ainda que os pacientes com dor mista apresentam quadro clínico mais complexo, com maior impacto na qualidade de vida e maior índice de subtratamento. Observamos, na nossa casuística, a presença de componente neuropático associado a dor em $39,5 \%$ dos pacientes.

Dos pacientes com componente de dor neuropática, foi observado que 8 de 17 pacientes (47\%) apresentaram pontuação no Quick-DASH $>50$, indicando pior resultado funcional, enquanto nos pacientes com DN $4<4$, apenas 4 entre 26 pacientes $(15,3 \%)$ apresentaram pontuação $>50$ no QuickDASH, sugerindo impacto da dor neuropática na reabilitação.

Na literatura, a dor neuropática geralmente está mais associada ao sexo feminino; ${ }^{12}$ no entanto, no presente estudo, não foi observada esta associação. Da mesma forma, não houve diferença estatisticamente significativa nos resultados do Quick-DASH entre os sexos, sugerindo que a lesão em si é mais importante do que o sexo no desenvolvimento do quadro de dor crônica.

Segundo a definição proposta por Freynhagen et al., ${ }^{13}$ a dor mista caracteriza-se pela sobreposição, em um mesmo segmento corpóreo, de dois ou mais mecanismos de dor (nociceptiva, neuropático ou nociplástico) e um ou outro mecanismo pode predominar ao longo do tempo, o que, por vezes, torna difícil identificar todos os componentes. 0 emprego de ferramentas específicas para avaliação de dor neuropática permite a identificação deste componente de forma simples e rápida.

Dentre os instrumentos disponíveis, o questionário DN4, desenvolvido na França por Bouhassira et al. ${ }^{14}$ em 2005 e traduzido e validado para o Brasil em 2011 por Santos et al., ${ }^{7}$ consiste em uma ferramenta para rastrear a dor neuropática, podendo ser utilizada tanto por especialistas quanto por não especialistas. O DN4 é composto por sete itens que se referem a sintomas e outros três que se relacionam com o exame físico. Cada item pontua um se a resposta for positiva e zero se negativa. Escores $\geq 4$ indicam presença de dor neuropática. Os resultados do estudo de validação da versão em português demonstraram sensibilidade de $100 \%$ e especificidade de $93,2 \%$.

No presente estudo, a pontuação inicial no DN4 $\geq 4$ foi fator determinante para a persistência da dor, confirmando a importância do componente neuropático da dor. A avaliação adequada, com diagnóstico precoce do componente neuropático de dor, associado ao componente nociceptivo, pode permitir o controle adequado da dor, impedindo sua cronificação e garantindo melhor reabilitação.

\section{Conclusão}

A prevalência de dor neuropática nos pacientes com fraturas da extremidade distal do rádio tratadas com placa volar bloqueada foi significativa, sendo de 39,5\% nesta casuística, e a presença de sinais e sintomas de dor neuropática foi fator preditivo positivo para a persistência da dor além de 2 meses em $100 \%$ dos casos.

\section{Suporte Financeiro}

Não houve suporte financeiro de fontes públicas, comerciais, ou sem fins lucrativos.

Conflito de Interesses

Os autores declaram não haver conflito de interesses.

\section{Referências}

1 Pires PR. Fraturas do rádio distal. In: Traumatologia Ortopédica. Rio de Janeiro: Revinter; 2004

2 Machado DG, Cerqueira SAC, Rodarte RR, Araújo Netto CA, Mathias MB. Statistical Analysis on Functional and Radiographic Results After Use of Locked Volar Plate for Fractures of the Distal Radius. Rev Bras Ortop 2012;47(03):297-303

3 Macrae WA. Chronic pain after surgery. Br J Anaesth 2001;87(01): 88-98

4 Kraychete DC, Sakata RK, Lannes LO, Bandeira ID, Sadatsune EJ. Postoperative persistent chronic pain: what do we know about prevention, risk factors and treatment. Rev Bras Anestesiol 2016; 66(05):505-512

5 Kehlet H, Jensen TS, Woolf CJ. Persistent postsurgical pain: risk factors and prevention. Lancet 2006;367(9522):1618-1625

6 Perkins FM, Kehlet H. Chronic pain as an outcome of surgery. A review of predictive factors. Anesthesiology 2000;93(04): 1123-1133

7 Santos JG, Brito JO, de Andrade DC, et al. Translation to Portuguese and validation of the Douleur Neuropathique 4 questionnaire. J Pain 2010;11(05):484-490

8 Beaton DE, Wright JG, Katz JN. Upper Extremity Collaborative Group. Development of the QuickDASH: comparison of three item-reduction approaches. J Bone Joint Surg Am 2005;87(05): 1038-1046

9 Orbay JL, Fernandez DL. Volar fixation for dorsally displaced fractures of the distal radius: a preliminary report. J Hand Surg Am 2002;27(02):205-215 
10 Catalano LW 3rd., Cole RJ, Gelberman RH, Evanoff BA, Gilula LA, Borrelli J Jr. Displaced intra-articular fractures of the distal aspect of the radius. Long-term results in young adults after open reduction and internal fixation. J Bone Joint Surg Am 1997;79 (09):1290-1302

11 Ibor PJ, Sánchez-Magro I, Villoria J, Leal A, Esquivias A. Mixed Pain Can Be Discerned in the Primary Care and Orthopedics Settings in Spain: A Large Cross-Sectional Study. Clin J Pain 2017;33(12):1100-1108

12 Roberto A, Deandrea S, Greco MT, et al. Prevalence of neuropathic pain in cancer patients: pooled estimates from a systematic review of published literature and results from a survey conducted in 50 Italian Palliative Care Centers. J Pain Symptom Manage 2016;51(06):1091-1102.e4

13 Freynhagen R, Parada HA, Calderon-Ospina CA, et al. Current understanding of the mixed pain concept: a brief narrative review. Curr Med Res Opin 2019;35(06):1011-1018

14 Bouhassira D, Attal N, Alchaar H, et al. Comparison of pain syndromes associated with nervous or somatic lesions and development of a new neuropathic pain diagnostic questionnaire (DN4). Pain 2005;114(1-2):29-36 